\title{
Automated Discovery of Detectors and Iteration-Performing Calculations to Recognize Patterns in Protein Sequences Using Genetic Programming
}

\author{
John R. Koza \\ Computer Science Department \\ Stanford University \\ Stanford, California 94305-2140 \\ Koza@CS.Stanford.Edu, 415-941-0336
}

\begin{abstract}
This paper describes an automated process for the dynamic creation of a pattern-recognizing computer program consisting of initially-unknown detectors, an initially-unknown iterative calculation incorporating the as-yet-uncreated detectors, and an initially-unspecified final calculation incorporating the results of the as-yetuncreated iteration. The program's goal is to recognize a given protein segment as being a transmembrane domain or non-transmembrane area. The recognizing program to solve this problem will be evolved using the recentlydeveloped genetic programming paradigm. Genetic programming starts with a primordial ooze of randomly generated computer programs composed of available programmatic ingredients and then genetically breeds the population using the Darwinian principle of survival of the fittest and the genetic crossover (sexual recombination) operation. Automatic function definition enables genetic programming to dynamically create subroutines (detectors). When cross-validated, the best genetically-evolved recognizer achieves an out-of-sample correlation of 0.968 and an outof-sample error rate of $1.6 \%$. This error rate is better than that recently reported for five other methods.
\end{abstract}

\section{Statement of the Problem}

The goal in this paper is to use genetic programming with automatically defined functions (ADFs) to create a computer program for recognizing a given subsequence of amino acids in a protein as being a transmembrane domain or non-transmembrane area of the protein. The automated process that will create the recognizing program for this problem will be given a set of differently-sized protein segments and the correct classification for each segment. The recognizing program will consist of initiallyunspecified detectors, an initially-unspecified iterative calculation incorporating the as-yet-undiscovered detectors, and an initially-unspecified final calculation incorporating the results of the as-yet-undiscovered iteration.

Although genetic programming does not know the chemical characteristics or biological meaning of the sequence of amino acids appearing in the protein segment, we will show that the results have an interesting biological interpretation. Of course, the reader may ignore the biological interpretation and view this problem as a onedimensional pattern recognition problem.

Genetic programming is a domain-independent method for evolving computer programs that solve, or approximately solve, problems. To accomplish this, genetic programming starts with a primordial ooze of randomly generated computer programs composed of the available programmatic ingredients, and breeds the population or programs using the Darwinian principle of survival of the fittest and an analog of the naturally occurring genetic operation of crossover (sexual recombination). Automatic function definition enables genetic programming to dynamically create subroutines dynamically during the run. The question arises as to whether genetic programming can evolve a recognizing program consisting of initially unspecified detectors, an initially unspecified iterative calculation incorporating the as-yet-undiscovered detectors, and an initially unspecified final calculation incorporating the results of the as-yet-undiscovered iteration. The genetically evolved program in this paper accomplishes this. It achieves a better error rate than all four algorithms described in Weiss et al. (1993). When analyzed, the genetically evolved program has a simple biological interpretation.

\section{Transmembrane Domains in Proteins}

Proteins are polypeptide molecules composed of sequences of amino acids. There are 20 amino acids (residues) in the alphabet of proteins. They are denoted by the letters $A, C$, $D, E, F, G, H, I, K, L, M, N, P, Q, R, S, T, V, W$, and $Y$. Broadly speaking, the sequence of amino acids in a protein determines the locations of its atoms in three-dimensional space; this, in turn, determines the biological structure and function of a protein (Anfinsen 1973).

A transmembrane protein is a protein that finds itself embedded in a membrane (e.g., a cell wall) in such a way 
that part of the protein is located on one side of the membrane, part is within the membrane, and part is on the opposite side of the membrane. Transmembrane proteins often cross back and forth through the membrane several times and have short loops immersed in the different milieu on each side of the membrane. The length of each transmembrane domain and each loop or other nontransmembrane area are usually different. Transmembrane proteins perform functions such as sensing the presence of certain chemicals or certain stimuli on one side of the membrane and transporting chemicals or transmitting signals to the other side of the membrane. Understanding the behavior of transmembrane proteins requires identification of their transmembrane domains.

Biological membranes are of hydrophobic (water-hating) composition. The amino acids in the transmembrane domain of a protein that are exposed to the membrane therefore have a pronounced tendency to be hydrophobic. This tendency toward hydrophobicity is an overall distributional characteristic of the entire segment (not of any particular few amino acids of the segment). Many transmembrane domains are $\alpha$-helices and our discussion here is limited to $\alpha$-helical transmembrane domains.

For example, a successful recognizing program should identify the following 24-residue segment from positions 96-119 of mouse peripheral myelin protein 22 (identified as "PM22_MOUSE" in the SWISS-PROT computerized database of proteins) as a transmembrane domain:

\section{(1) FYITGFFQILAGLCVMSAAAIYTV,}

Conversely, a successful program should identify the following 27-residue segment between positions $35-61$ as being in a non-transmembrane area of the protein:

\section{(2) TTDLWQNCTTSALGAVQHCYSSSVSEW}

This classification problem will be solved by genetic programming without reference to any knowledge about the hydrophobicity of the 20 amino acids; however, we will use such knowledge to explain the problem (and, later, to interpret the genetically evolved program). Two thirds of the 24 residues of segment (1) are in the category consisting of I, V, L, F, C, M, or A having the highest numerical values of hydrophobicity on Kyte-Doolittle scale. If a human were clustering the 20 hydrophobicity values into three categories with the benefit of knowledge of the Kyte-Doolittle hydrophobicity scale, these seven residues would be categorized into a hydrophobic category. Seven of the 24 residues of segment (1) (i.e., two Gs, two Ts, two $Y_{s}$, and one S) are in the category consisting of G, T, S, W, Y, P (which the knowledgeable human would cluster into a neutral category). Only one residue of segment (1) (i.e., the $Q$ at position 103) is in the category consisting of $H, Q, N, E, D, K, R$ (which the knowledgeable human would cluster into a hydrophilic category). Even through there are some residues from all three categories in segments(1), segment (1) is predominantly hydrophobic and is, in fact, a transmembrane domain of PM22_MOUSE.

In contrast, 13 of the 27 (about half) of the residues of segment (2) are neutral, eight (about a quarter) are hydrophobic, and six (about a quarter) are hydrophilic. This distribution is very different from that of segment (1). Segment (2) is, in fact, a non-transmembrane area.

\section{Background on Genetic Programming}

John Holland's pioneering Adaptation in Natural and Artificial Systems (1975, 1992) described how the evolutionary process in nature can be applied to artificial systems using the genetic algorithm operating on fixed length character strings.

Genetic programming is an extension of the genetic algorithm in which the genetic population consists of computer programs (that is, compositions of primitive functions and terminals). As described in Genetic Programming: On the Programming of Computers by Means of Natural Selection (Koza 1992), genetic programming is a domain independent method that genetically breeds populations of computer programs to solve problems by executing the following three steps:

(1) Generate an initial population of random computer programs composed of the primitive functions and terminals of the problem.

(2) Iteratively perform the following sub-steps until the termination criterion has been satisfied:

(a) Execute each program in the population and assign it a fitness value according to how well it solves the problem.

(b) Create a new population of programs by applying the following two primary operations. The operations are applied to program(s) in the population selected with a probability based on fitness (i.e., the fitter the program, the more likely it is to be selected).

(i) Reproduction: Copy an existing program to the new population.

(ii) Crossover: Create two new offspring programs for the new population by genetically recombining randomly chosen parts of two existing programs. The genetic crossover (sexual recombination) operation (described below) operates on two parental computer programs and produces two offspring programs using parts of each parent.

(3) The single best computer program in the population produced during the run is designated as the result of the run of genetic programming. This result may be a solution (or approximate solution) to the problem.

Recent advances in genetic programming are described in Kinnear (1994). A videotape visualization of numerous applications of genetic programming can be found in Koza and Rice (1992) and Koza and Rice (1994). 


\subsection{Crossover}

The genetic crossover operation operates on two parental computer programs selected with a probability based on fitness and produces two new offspring programs consisting of parts of each parent.

For example, consider the following computer program (shown here as a LISP symbolic expression):

$(+\underline{(* 0.234 \mathrm{Z})}(-\mathrm{X} 0.789))$.

We would ordinarily write this LISP S-expression as $0.234 z+x-0.789$. This two-input, one-output computer program takes $\mathrm{X}$ and $\mathrm{Z}$ as inputs and produces a single floating point output.

Also, consider a second program:

$(*(* \mathrm{Z} Y)(+\mathrm{Y}(* 0.314 \mathrm{Z})))$.

This program is equivalent to $z y(y+0.314 z)$.

The crossover operation creates new offspring by exchanging sub-trees (i.e., subroutines, sublists, subprocedures, subfunctions) between the two parents. The two parents are typically of different sizes and shapes. The sub-trees to be exchanged (called crossover fragments) are selected at random by selecting crossover points at random. Suppose that crossover points are the multiplication $(*)$ in the first parent and the addition $(+)$ in the second parent. The two crossover fragments are the underlined sub-programs (sub-lists) in the two parents.

The two offspring resulting from crossover are

$(+(+\mathrm{Y}(* 0.314 \mathrm{Z}))(-\mathrm{X} 0.789))$ and

$(*(* \mathrm{ZY})(* 0.234 \mathrm{Z}))$

Assuming closure of the functions and terminals of which the programs are composed, crossover produces syntactically and semantically valid programs as offspring.

\subsection{Automatic Function Definition}

Automatic function definition is used to enable genetic programming to evolve subroutines during a run. Automatic function definition can be implemented within the context of genetic programming by establishing a constrained syntactic structure for the individual programs in the population as described in Genetic Programming II: Scalable Automatic Programming by Means of Automatically Defined Functions (Koza 1994). Each program in the population contains one (or more) functiondefining branches, one main result-producing branch, and possibly other types of branches (such as iterationperforming branches). The function-defining branch(es) define the automatically defined functions $\mathrm{ADF} 0, \mathrm{ADF} 1$, etc. The result-producing branch may invoke the ADFs. The value returned by the overall program is the value returned by the result-producing branch.

The initial random generation of the population (generation 0) is created so that every individual program in the population has a constrained syntactic structure consisting of the problem's particular arrangement of branches. Each branch is composed of functions and terminals appropriate to that branch. This constrained syntactic structure must be preserved as the run proceeds from generation to generation. Structure-preserving crossover is implemented by limiting crossover to points lying within the bodies of the various branches (branch typing). The crossover point for the first parent is randomly selected, without restriction, from the body of any one of the branches. However, once this selection is made for the first parent, the crossover point of the second parent is randomly selected from the body from the same type of branch. This method of performing crossover preserves the syntactic validity of all offspring throughout the run. As the run progresses, genetic programming will evolve different function-defining branches, different result-producing branches, and different ways of calling these automatically defined functions from the resultproducing branch.

\section{Preparatory Steps}

In applying genetic programming with automatic function definition to a problem, there are six major preparatory steps. These steps involve determining

(1) the set of terminals for each branch,

(2) the set of functions for each branch,

(3) the fitness measure,

(4) the parameters and variables for controlling the run,

(5) the result designation and termination method and

(6) the architecture of the overall program.

\subsection{Architecture of the Overall Program}

We begin by deciding that the overall architecture of the yet-to-be-evolved recognizing program will have to be capable of categorizing the residues into useful categories, then iteratively performing some arithmetic calculations and conditional operations on the categories, and finally performing some arithmetic calculations and conditional operations to reach a conclusion. This suggests an overall architecture for the recognizing program of several automatically defined functions (say $\mathrm{ADF} 0, \mathrm{ADF} 1, \mathrm{ADF} 2$ ) to serve as detectors for categorization, an iterationperforming branch, IPBO, for performing arithmetic operations and conditional operations for examining the residues of the protein segment using the as-yetundiscovered detectors, and a result-producing branch, $\mathrm{RPBO}$, for performing arithmetic and conditional operations for reaching a conclusion.

\subsection{Function-Defining Branches}

Automatically defined functions seem well suited to the role of dynamically defining categories of the amino acids. If the automatically defined functions are to play the role of set formation, each defined function should be able to interrogate the current residue as to which of the 20 amino acids it is. Since we anticipate that some numerical calculations will subsequently be performed on the result of the categorization of the residues, we employ numericalvalued logic, rather than Boolean-valued logic returning the non-numerical values of True and False. One way to 
implement this approach is to define 20 numerical-valued zero-argument logical functions for determining whether the residue currently being examined is a particular amino acid. For example, (A?) is the zero-argument residuedetecting function returning a numerical +1 if the current residue is alanine $(A)$ but otherwise returning a numerical -1 . A similar residue-detecting function is defined for each of the 19 other amino acids. Since we envisage that the automatically defined functions will be used for set formation, it seems reasonable to include the logical disjunctive function in the function set of the automatically defined functions. ORN is the two-argument numericalvalued disjunctive function returning +1 if either or both of its arguments are positive, but returning -1 otherwise.

The terminal set for each of the three function-defining branches (ADF 0, $A D F 1$, and $A D F 2$ ) contains the 20 zeroargument numerical-valued residue-detecting functions.

$$
\tau_{\mathrm{fd}}=\{(\mathrm{A} ?),(\mathrm{C} ?), \ldots,(\mathrm{Y} ?)\} .
$$

The function set for the three function-defining branches (ADF 0, ADF 1, and ADF2) contains only the two-argument numerically-valued logical disjunctive function.

$F_{\mathrm{fd}}=\{\mathrm{ORN}\}$.

\subsection{Iteration-Performing Branch}

Typical computer programs contain iterative operators that perform some specified work until some condition expressed by a termination predicate is satisfied. When we attempt to include iterative operators in geneticallyevolved programs, we face the practical problem that both the work and the termination predicate are initially created at random and are subsequently subject to modification by the crossover operation. Consequently, iterative operators will, at best, be nested and consume enormous amounts of computer time or will, at worst, have unsatisfiable termination predicates and go into infinite loops. This problem can sometimes be partially alleviated by imposing arbitrary time-out limits (e.g., on each iterative loop individually and all iterative loops cumulatively).

In problems where we can envisage one iterative calculation being usefully performed over a particular known, finite set, there is an attractive alternative to permitting imposing arbitrary time-out limits. For such problems, the iteration can be restricted to exactly one iteration over the finite set. The termination predicate of the iteration is thereby fixed and is not subject to evolutionary modification. Thus, there is no nesting and there are no infinite loops.

In the case of problems involving the examination of the residues of a protein, iteration can very naturally be limited to the ordered set of amino acid residues of the protein segment involved. Thus, for this problem, we employ one iteration-performing branch, with the iteration restricted to the ordered set of amino acid residues in the protein segment. That is, each time iterative work is performed by the body of the iteration-performing branch, the current residue of the protein is advanced to the next residue of the protein segment until the end of the entire protein segment is encountered. The result-producing (wrap-up) branch produces the final output of the overall program.

Useful iterative calculations typically require both an iteration variable and memory (state). That is, the nature of the work performed by the body of the iterationperforming branch typically varies depending on the current value of the iteration variable. Memory is typically required to transmit information from one iteration to the next. In this problem, the same work is executed as many times as there are residues in a protein segment, so the iteration variable is the residue at the current position in the segment. Depending on the problem, the iteration variable may be explicitly available or be implicitly available through functions that permit it to be interrogated. For this problem, the automatically defined functions provide a way to interrogate the residues of the protein sequence.

Memory can be introduced into any program by means of settable variables, M0, M1, M2, and M3. Settable variables are initialized to some appropriate value (e.g., zero) at the beginning of the execution of the iteration-performing branch. These settable variables typically change as a result of each iteration.

The terminal set for the iteration-performing branch is

$\tau_{\text {ipb0 }}=\{\mathrm{LEN}, \mathrm{M} 0, \mathrm{M} 1, \mathrm{M} 2, \mathrm{M} 3, \leftarrow\}$.

Here $\leftarrow$ represents floating-point random constants between -10.000 and +10.000 with a granularity of 0.001 and LEN is the length of the current protein segment.

Since we envisage that the iteration-performing branch will perform numerical calculations and make decisions based on these calculations, it seems reasonable to include the four arithmetic operations and a conditional operator in the function set. We have used the four arithmetic functions $\left(+,-,{ }^{*}\right.$, and $\left.\stackrel{\circ}{\circ}\right)$ and the conditional comparative operator IFLTE (If Less Than or Equal) on many previous problems, so we include them in the function set for the iteration-performing branch. The protected division function $\%$ takes two arguments and returns one when division by 0 is attempted (including 0 divided by 0 ), and, otherwise, returns the normal quotient. The four-argument conditional branching function IFLTE evaluates and returns its third argument if its first argument is less than or equal to its second argument and otherwise evaluates and returns its fourth argument.

Since a numerical calculation is to be performed on the results of the categorization performed by the functiondefining branches, the functions $\mathrm{ADF} 0, \mathrm{ADF} 1$, and $\mathrm{ADF} 2$ are included in the function set for the iteration-performing branch.

We need a way to change the settable variables M0, M1, $\mathrm{M} 2$, and M3. The one-argument setting function SETMO can be used to set MO to a particular value. Similarly, the 
setting functions SETM1, SETM2, and SETM3 can be used to set the respective values of the settable variables $M 1$, M2, and M3, respectively. Thus, memory can be written (i.e., the state can be set) with the setting functions, SETM0, SETM1, SETM2, and SETM3, and memory can be read (i.e., the state can be interrogated) merely by referring to the terminals, M0, M1, M2, and M3.

Thus, the function set for the iteration-performing branch, IPBO, is

$$
\begin{gathered}
F_{\text {ipb0 }}=\{\operatorname{ADF} 0, \operatorname{ADF} 1, \operatorname{ADF} 2, \operatorname{SETM} 0, \operatorname{SETM} 1, \\
\text { SETM2, SETM3, IFLTE, +, - * * 은 }\} . \\
\text { taking } 0,0,0,1,1,1,1,4,2,2,2 \text {, and } 2 \text { arguments. }
\end{gathered}
$$

\subsection{Result-Producing Branch}

The result-producing (wrap-up) branch then performs a non-iterative floating-point calculation and produces the final result of the overall program. The settable variables M0, M1, M2, and M3 provide a way to pass the results of the iteration-performing branch to the result-producing branch.

The terminal set for the result-producing branch, RPBO, is

$\tau_{\mathrm{rpb} 0}=\{\mathrm{LEN}, \mathrm{M} 0, \mathrm{M} 1, \mathrm{M} 2, \mathrm{M} 3, \leftarrow\}$.

The function set for the result-producing branch RPBO, is

$F_{\text {rpb0 }}=\left\{\right.$ IFLTE,,+- ,,$\left.\frac{\circ}{\circ}\right\}$

taking 4, 2, 2, 2, and 2 arguments, respectively.

A wrapper is used to convert the floating-point value produced by the result-producing branch into a binary outcome. If the genetically-evolved program returns a positive value, the segment will be classified as a transmembrane domain, but otherwise it will be classified as a non-transmembrane area.

\subsection{Fitness Cases}

Release 25 of the SWISS-PROT protein data base (Bairoch and Boeckmann 1991) contains 248 mouse transmembrane proteins averaging 499.8 residues in length. Each protein contains between one and 12 transmembrane domains, the average being 2.4. The transmembrane domains range in length from 15 and 101 residues and average 23.0.

123 of the 248 proteins were arbitrarily selected to create the in-sample set of fitness cases to measure fitness during the evolutionary process. One of the transmembrane domains of each of these 123 proteins was selected at random as a positive fitness case for this in-sample set. One segment of the same length as a random one of the transmembrane segments that is not contained in any of the protein's transmembrane domains was selected from each protein as a negative fitness case. There are 123 positive and 123 negative fitness cases in the in-sample set.

The evolutionary process is driven by fitness as measured by the set of in-sample fitness cases. However, the true measure of performance for a recognizing program is how well it generalizes to different cases from the same problem environment. Thus, 250 out-of-sample fitness cases (125 positive and 125 negative) were created from the remaining 125 proteins in a manner similar to the above. These out-of-sample fitness cases were then used to validate the performance of the genetically-evolved programs.

\subsection{Fitness Measure}

Fitness will measure how well a particular geneticallyevolved recognizing program predicts whether the segment is, or is not, transmembrane domain. Fitness is measured over a number of trials, which we call fitness cases. The fitness cases for this problem consist of protein segments.

When a genetically-evolved recognizing program in the population is tested against a particular fitness case, the outcome can be a true-positive, true-negative, falsepositive, or false-negative. Fitness can be measured by the correlation coefficient $C$. When the predictions and observations each take on only two possible values, correlation is a general, and easily computed, measure for evaluating the performance of a recognizing program.

The correlation, $C$, lends itself immediately to being the measure of raw fitness measure for a genetically evolved computer program. Since raw fitness ranges between -1.0 and +1.0 (higher values being better), standardized fitness ("zero is best") can then be defined as $\frac{1-C}{2}$. Standardized fitness ranges between 0.0 and +1.0 , lower values being better and a value of 0 being the best. A standardized fitness of 0 indicates perfect agreement between the predicting program and the observed reality; a standardized fitness of +1.0 indicates total disagreement; 0.50 indicates that the predictor is no better than random.

The error rate is the number of fitness cases for which the recognizing program is incorrect divided by the total number of fitness cases. The error rate is a less general measure of performance for a recognizing program; however, Weiss et al. (1993) use the error rate as their yardstick for comparing three methods in the biological literature with their new algorithm created using the SWAP-1 induction technique. Therefore, we present our final results in terms of both correlation and error rate and we use error rate for the purpose of comparing results.

\subsection{Control Parameters}

Population size, $M$, was 4,000 . The maximum number of generations to be run, $G$, was set to 21 . The other parameters for controlling the runs of genetic programming were the default values specified in Koza (1994) and which have been used for a number of different problems.

\section{Results}

The best program over 11 runs produced a value of out-ofsample correlation of 0.968 on generation 20 . The insample correlation of 0.976 results from 121 true positives, 122 true negatives, 1 false positive, and 2 false negatives over the 246 in-sample fitness cases. The out-of-sample correlation of 0.968 is the result of 123 true positives, 123 
true negatives, 2 false positives, and 2 false negatives . The out-of-sample error rate is only $1.6 \%$. This 105 -point best program is shown below:

(progn (defun ADFO ()

(values (ORN (ORN (ORN (I?) (H?)) (ORN (P?)

(G?))) (ORN (ORN (ORN (Y?) (N?)) (ORN (T?)

$(\mathrm{Q} ?)))(\mathrm{ORN}(\mathrm{A} ?)(\mathrm{H} ?))))))$

(defun ADF1 ()

(values (ORN (ORN (ORN (A?) (I?)) (ORN (L?)

(W?))) (ORN (ORN (T?) (L?)) (ORN (T?)

$(\mathrm{W} ?)))))$ )

(defun ADF2 ()

(values (ORN (ORN (ORN (ORN (ORN (D?) (E?))

(ORN (ORN (ORN (D?) (E?)) (ORN (ORN (T?)

(W?)) (ORN (Q?) (D?)))) (ORN (K?) (P?)))) (ORN

(K?) (P?))) (ORN (T?) (W?))) (ORN (ORN (E?)

(A?)) (ORN (N?) (R?))))))

(progn (loop-over-residues (SETM0 (+ (- (ADF1)

(ADF2)) (SETM3 M0))))

(values (\% (\% M3 M0) $(\%$ (\% (\% (- L - 0.53$)(*$

M0 M0)) (+ (\% (\% M3 M0) (\% (+ M0 M3) (\% M1

M2))) M2)) (\% M3 M0))))))

Ignoring the three residues common to the definition of both $A D F 1$ and $A D F 2, A D F 1$ returns 1 if the current residue is $I$ or $L$ and $A D F 2$ returns 1 if the current residue is $D, E, K, R, Q, N$, or $P$. I and $L$ are two of the seven hydrophobic residues on the Kyte-Doolittle scale. D, E, K, $R, Q$, and $N$ are six of the seven hydrophilic residues, and $P$ is one of the neutral residues.

In the iteration-performing branch of this program from generation 20, M0 is the running sum of the differences of the values returned by $A D F 1$ and $A D F 2$. MO will be positive only if the hydrophobic residues in the protein segment are so numerous that the occurrences of $I$ and $L$ outnumber the occurrences of the six hydrophilic residues and one neutral residue of $A D F 2 . M 3$ is the same as the accumulated value of MO except that M3 lags M0 by one residue. Because the contribution to M3 in the iterationperforming branch of the last residue is either 0 or $1, \mathrm{M} 3$ is either equal to $\mathrm{M} 0$ or is one less than $\mathrm{M} 0$.

The result-producing branch is equivalent to

$$
\frac{M_{3}^{3}}{M_{0}\left(M_{0}+M_{3}\right)(\text { Len }+0.53)}
$$

The subexpression ( - LEN -0.53 ) is always positive and therefore can be ignored in determining whether the result-producing branch is positive or nonpositive. Because of the close relationship between M0 and M3, analysis shows that the result-producing branch identifies a protein segment as a transmembrane domain whenever the running sum of the differences, MO, is greater than 0 , except for the special case when $\mathrm{MO}=1$ and $\mathrm{M} 3=0$. This special case occurs only when the running values of $\mathrm{M} 0$ and M3 are tied at 0 and when the very last residue of the protein segment is I or $L$ (i.e., $A D F 1$ returns 1 ).
Ignoring this special case, we can summarize the operation of this overall best-of-all program from generation 20 as follows: If the number of occurrences of $I$ and $L$ in a given protein segment exceeds the number of occurrences of $D$, $E, K, R, Q, N$, and $P$, classify the segment as a transmembrane domain; otherwise, classify it as a nontransmembrane area.

\section{Conclusions}

Table 1 shows the out-of-sample error rate for the four algorithms for recognizing transmembrane domains reviewed in Weiss et al. (1993) as well as the out-ofsample error rate of our best-of-all genetically-evolved program above. We wrote a computer program to test the solution discovered by the SWAP-1 induction technique used in the first experiment of Weiss et al. (1993). Our implementation of their solution produced an error rate on our test data identical to the error rate reported by them on their own test data (i.e., the $2.5 \%$ of row 4 of the table).

As can be seen, the error rate of the best-of-all geneticallyevolved program from generation 20 is better than the error rates of the other four methods reported in the table. This genetically evolved program is an instance of an algorithm discovered by an automated learning paradigm that is superior to that written by human investigators.

In summary, without using foreknowledge of hydrophobicity, genetic programming with automatic function definition was able to evolve a successful recognizing program consisting of initially-unspecified detectors, an initially-unspecified iterative calculation incorporating the as-yet-undiscovered detectors, and an initially-unspecified final calculation incorporating the results of the as-yet-undiscovered iteration.

Table 1 Comparison of five methods.

\begin{tabular}{|l|l|}
\hline Method & Error rate \\
\hline von Heijne 1992 & $2.8 \%$ \\
\hline Engelman et al. 1986 & $2.7 \%$ \\
\hline Kyte-Doolittle 1982 & $2.5 \%$ \\
\hline Weiss et al. 1993 & $2.5 \%$ \\
\hline Best evolved program & $1.6 \%$ \\
\hline
\end{tabular}

7. Acknowledgements

James P. Rice of the Knowledge Systems Laboratory at Stanford University did the computer programming of the above on a Texas Instruments Explorer $\mathrm{II}^{+}$computer.

\section{Bibliography}

Anfinsen, C. B. 1973. Principles that govern the folding of protein chains. Science 81: 223-230.

Bairoch, A. and Boeckmann, B. 1991. The SWISS PROT protein sequence data bank. Nucleic Acids Research 19:2247-2249.

Engelman, D., Steitz, T., and Goldman, A. 1986. Identifying nonpolar transbilayer helices in amino acid sequences of membrane proteins. Annual Review of Biophysics and Biophysiological Chemistry. Volume 15. 
Holland, J. H. Adaptation in Natural and Artificial Systems: An Introductory Analysis with Applications to Biology, Control, and Artificial Intelligence. University of Michigan Press 1975. Also available from The MIT Press 1992.

Kinnear, K. E. Jr. (editor). 1994. Advances in Genetic Programming. The MIT Press.

Koza, J. R. 1992. Genetic Programming: On the Programming of Computers by Means of Natural Selection. The MIT Press.

Koza, J. R. 1994. Genetic Programming II. The MIT Press.

Koza, J. R., and Rice, J. P. 1992.Genetic Programming: The Movie. The MIT Press.

Koza, J. R., and Rice, J. P. 1994. Genetic Programming II Videotape: The Next Generation. The MIT Press.

Kyte, J. and Doolittle, R. 1982. A simple method for displaying the hydropathic character of proteins. Journal of Molecular Biology. 157:105-132.

von Heijne, G. Membrane protein structure prediction: Hydrophobicity analysis and the positive-inside rule. Journal of Molecular Biology. 225:487-494.

Weiss, S. M., Cohen, D. M., and Indurkhya, N. 1993. Transmembrane segment prediction from protein sequence data. In Hunter, L., Searls, D., and Shavlik, J. (editors). Proceedings of the First International Conference on Intelligent Systems for Molecular Biology. AAAI Press. 